\title{
Spin-layer locking of interlayer excitons trapped in moiré potentials
}

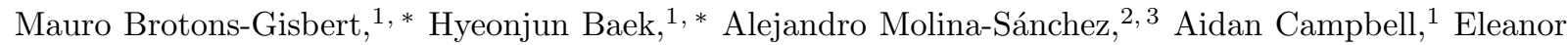 \\ Scerri, ${ }^{1}$ Daniel White, ${ }^{1}$ Kenji Watanabe, ${ }^{4}$ Takashi Taniguchi, ${ }^{4}$ Cristian Bonato, ${ }^{1}$ and Brian D. Gerardot ${ }^{1, \dagger}$ \\ ${ }^{1}$ Institute of Photonics and Quantum Sciences, SUPA, Heriot-Watt University, Edinburgh EH14 4AS, UK \\ ${ }^{2}$ Institute of Materials Science (ICMUV), University of Valencia, Catedrático Beltrán 2, E-46980 Valencia, Spain \\ ${ }^{3}$ International Iberian Nanotechnology Laboratory (INL), Avda. Mestre José Veiga s/n, 4715-330 Braga, Portugal \\ ${ }^{4}$ National Institute for Materials Science, Tsukuba, Japan
}

(Dated: June 3, 2020)

\begin{abstract}
Van der Waals heterostructures offer attractive opportunities to design quantum materials. For instance, transition metal dichalcogenides (TMDs) possess three quantum degrees of freedom: spin, valley index, and layer index. Further, twisted TMD heterobilayers can form moiré patterns that modulate the electronic band structure according to atomic registry, leading to spatial confinement of interlayer exciton (IXs). Here we report the observation of spin-layer locking of IXs trapped in moiré potentials formed in a heterostructure of bilayer $2 \mathrm{H}-\mathrm{MoSe}_{2}$ and monolayer $\mathrm{WSe}_{2}$. The phenomenon of locked electron spin and layer index leads to two quantum-confined IX species with distinct spin-layer-valley configurations. Furthermore, we observe that the atomic registries of the moiré trapping sites in the three layers are intrinsically locked together due to the $2 \mathrm{H}$-type stacking characteristic of bilayer TMDs. These results identify the layer index as a useful degree of freedom to engineer tunable few-level quantum systems in two-dimensional heterostructures.
\end{abstract}

The electronic and optical properties of van der Waals heterostructures can be widely engineered by the diverse choice of crystal combinations 1 and their relative rotation [2, 6] and interlayer spacing [7, 8]. For nearly commensurate bilayers (BLs), a slight lattice mismatch or relative rotation results in a moiré superlattice which spatially modulates the electronic band-structure. Single particle wavepackets can be trapped in the moiré-induced potential pockets with three-fold symmetry [9 11. Recently, signatures of IX trapped in such moiré potentials were observed in TMD hetero-BL samples [12, 13].

Due to the potential to harness the carrier spin, valley index, and layer index [14, layered TMDs present an intriguing platform for quantum electronics and optics. The broken inversion symmetry in TMDs leads to an effective coupling between a carrier spin and the valley index of the electrons or holes at the $\pm \mathrm{K}$ corners of the hexagonal Brillouin zone [15, as shown for monolayer (ML) $\mathrm{WSe}_{2}$ in Fig. 11. Like the real spin, the valley index is associated to a magnetic moment [14, 15], which results in valley dependent selection rules. Light with $\sigma^{ \pm}$-polarisation creates electron-hole pairs exclusively in the $\pm \mathrm{K}$ valley, enabling optical and magnetic manipulation of intralayer valley excitons [16-19]. Layer index is found in $2 H$-type BL TMDs which have a $180^{\circ}$ in-plane rotation between the top- and bottom-layer and minimal interlayer electronic hopping at the $\mathrm{K}$ valleys [15, 20,22]. The in-plane rotation inverts the valley alignment in the two layers, such that a unique spin-valley configuration is locked to each layer, as shown for BL $\mathrm{MoSe}_{2}$ in Fig. 1k. This gives rise to the layer index. Additionally, the atomic registries of the two layers are intrinsically locked together in $2 H$-type BL TMDs.
Owing to these quantum degrees of freedom, TMDs are ideal ingredients to realise the concept of exciton trapping in moiré potentials 913. Two different ML TMDs can be combined with an atomically sharp interface to create a hetero-BL system with Type II-band alignment [23. 24], which favours spatial separation of photogenerated carriers: electrons and holes rapidly transfer to reside in the layer with the lowest energy conduction (CB) and valence band-edge (VB), respectively [25. Due to strong Coulomb interaction, the electrons and holes form spatially-indirect interlayer excitons which exhibit the valley-dependent optical selection rules of the monolayer TMDs but with longer exciton lifetimes, robust spin-valley polarisation, and large electric field tunability 26 30. Hetero-BLs with arbitrary stacking angles can be fabricated by transfer of mechanically exfoliated flakes [12, 13, 24, 27, 30 or chemical vapour deposition [3, 23, 25, 29. Nearly aligned $0^{\circ}$ ( $R$-type) or $60^{\circ}(H$ type) stacking of the hetero-BL yields minimum displacement in momentum space for carriers at the band edges $( \pm \mathrm{K})$, maximizing coupling to the light cone for optical transitions [26. As shown in Fig. 1 $\mathrm{d}$, IXs in an $R$-type stacked hetero-BL $\left(\mathrm{IX}^{R}\right)$ have valley conserving ground state optical transitions, identical to monolayer TMDs. Conversely, IXs in $H$-type stacked materials $\left(\mathrm{IX}^{H}\right.$ ) have valley contrasting optical transitions. We label the bandedge states at $\pm \mathrm{K}$ as $|V, \uparrow / \downarrow\rangle,|L, \uparrow / \downarrow\rangle$, and $|U, \uparrow / \downarrow\rangle$, where $V$ represents the $\mathrm{WSe}_{2}$ valence band, $U(L)$ is the upper (lower) $\mathrm{MoSe}_{2}$ layer (layer index), and $\uparrow(\downarrow)$ represents the electron spin $s_{z}=1 / 2(-1 / 2)$. In addition, nearly aligned $0^{\circ}$ or $60^{\circ}$ stacking yields a moiré potential landscape in a hetero-BL in which three trapping sites $\left(\mathrm{A}, \mathrm{B}\right.$, and C) with atomic registries $R_{h}^{h}(\mathrm{~A}), R_{h}^{X}(\mathrm{~B})$ 

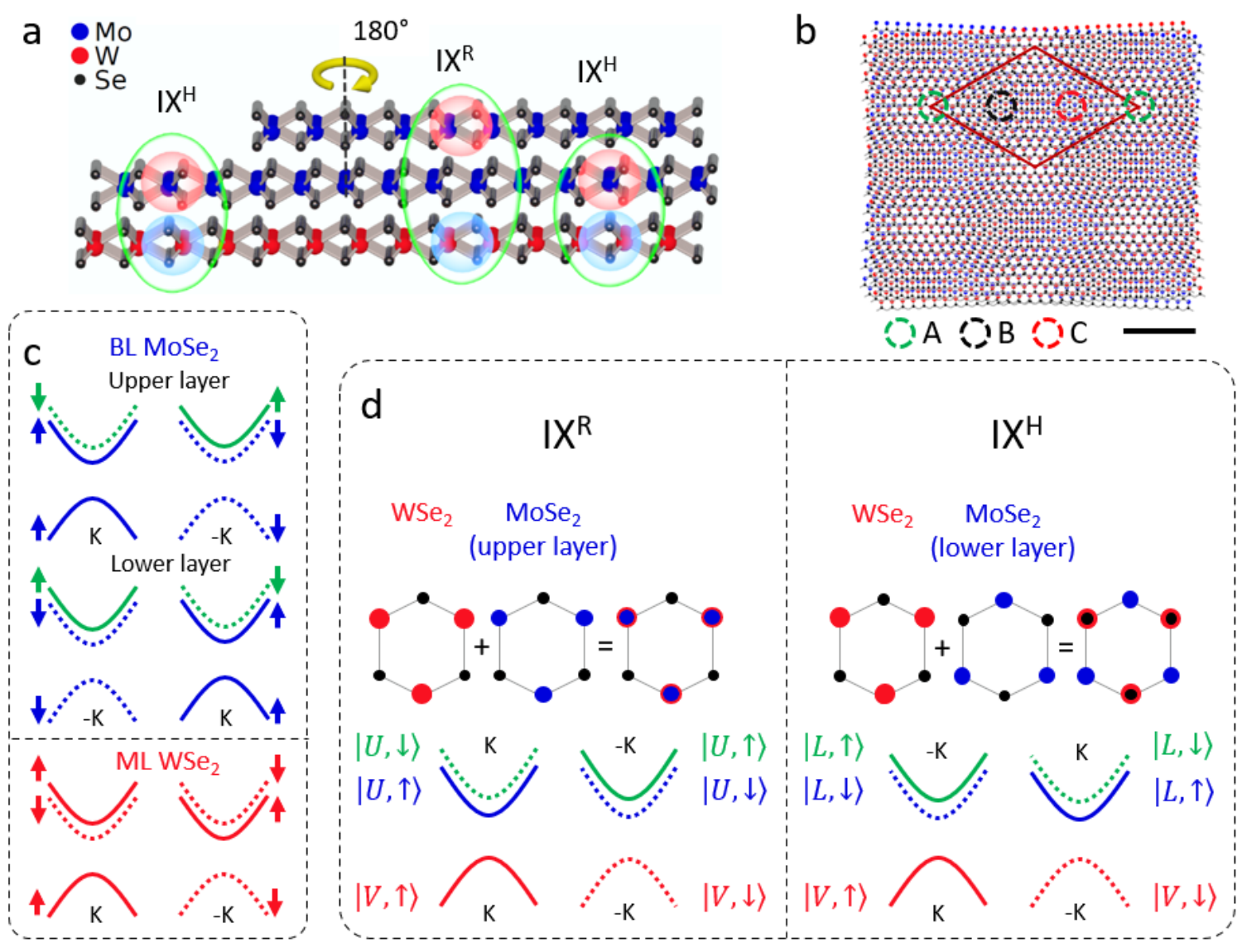

FIG. 1: Spin-layer locking of moiré-trapped IXs in a van der Waals heterostructure. a, Sketch of the heterostructures used in this work: a $2 H-\mathrm{MoSe}_{2}$ crystal with ML- and BL-thick terraces stacked on top of a $\mathrm{WSe}_{2} \mathrm{ML}$. The yellow arrow represents the $2 H$ stacking of $\mathrm{BL} \mathrm{MoSe}_{2}$. The red, blue and black dots represent the $\mathrm{W}$, Mo, and Se atoms, respectively. The relative twists between the $\mathrm{WSe}_{2}$ and the top and bottom $\mathrm{MoSe}_{2}$ layers are $\sim 0^{\circ}$ ( $3 R$ stacking) and $\sim 60^{\circ}(2 H$ stacking), respectively. Electrons (red shadows) at the $\pm \mathrm{K}$-valleys, localised either in the bottom or top layer $\mathrm{MoSe}_{2}$, are strongly bound to holes (blue shadows) in the $\mathrm{WSe}_{2}$, creating two species of IX (green circles): IX ${ }^{H}$ and $\mathrm{IX}^{R}$, respectively. $\mathbf{b}$, Cartoon of the moiré superlattice formed in a $R$-stacked $\mathrm{WSe}_{2} / \mathrm{MoSe}_{2}$ heterostructure with a twist angle $\theta$ of $5^{\circ}$. Scale bar, $2 \mathrm{~nm}$. The red diamond represents a moiré supercell. The three highlighted regions represent moiré-trapping sites with different local atomic configurations: A $\left(R_{h}^{h}\right), \mathrm{B}\left(R_{h}^{X}\right)$ and $\mathrm{C}\left(R_{h}^{M}\right)$. c, Spin-valley locked configuration of BL $2 \mathrm{H}-\mathrm{MoSe}_{2}$ (top panel) and $\mathrm{ML} \mathrm{WSe}_{2}$ (bottom panel) at the $\pm \mathrm{K}$ corners of the hexagonal Brillouin zone. The red lines represent the electronic band structure at the direct band-gap edges of $\mathrm{WSe}_{2}$. The blue lines denote the valence and conduction band-edges of $\mathrm{MoSe}_{2}$, while the green lines represent the $\mathrm{MoSe}_{2} \mathrm{CB}$ excited state arising from spin-orbit coupling. The solid (dotted) lines depict spin-up (down) band-edge states. Up (down) arrows indicate spin-up (down) conduction-band and valence-band electrons. d, Valley- and spin-layer pairing of the electrons for IX ${ }^{R}$ (left) and IX ${ }^{H}$ (right) together with the top views of the corresponding stacking configurations. The labels $|V, \uparrow / \downarrow\rangle,|L, \uparrow / \downarrow\rangle$, and $|U, \uparrow / \downarrow\rangle$ represent the band-edge states at $\pm \mathrm{K}$, with $V$ the $\mathrm{WSe}_{2}$ valence band, $U(L)$ the upper (lower) MoSe 2 layer (layer index), and $\uparrow(\downarrow)$ the electron spin $s_{z}=1 / 2(-1 / 2)$. The red, blue and black dots represent the atoms in the $\mathrm{WSe}_{2}$ and $\mathrm{MoSe}_{2}$ layers according to a.

and $R_{h}^{M}$ (C) emerge (see Fig. 1 $\mathrm{b}$ ), where $R_{h}^{\mu}$ denotes an $R$-type stacking with the $\mu$ site of the electron layer (either $h$ the hexagon centre, $X$ the chalcogen site or $M$ the metal site) vertically aligned with the hexagon centre $(h)$ of the hole layer [7, 9, 11.

Here we explore the spin, valley index, and layer index properties of moiré-trapped IXs in an artificial trilayer (TL) heterostructure consisting of a ML $\mathrm{WSe}_{2}$ and $\mathrm{BL}$ $2 \mathrm{H}$-MoSe ${ }_{2}$ (see Fig. 11). Due to the $d_{z^{2}}$ symmetry of the orbitals at the conduction band edge, electrons in $\mathrm{BL}$
$\mathrm{MoSe}_{2}$ present a vanishing interlayer hopping at the $\pm \mathrm{K}$ points, which leads to a strong coupling between the electron spin, the layer and the valley degrees of freedom [20. We exploit the spin-layer locking phenomenon of BL $2 \mathrm{H}$ $\mathrm{MoSe}_{2}$ to probe two moiré-trapped IX species with contrasting spin-valley alignment: the holes, localised in the $\mathrm{WSe}_{2}$ monolayer, are strongly Coulomb bound to electrons localised in either the lower or upper $\mathrm{MoSe}_{2}$ layer to form IX ${ }^{H}$ or IX ${ }^{R}$ species, respectively. Each localised IX species has a distinct spin-layer-valley configuration: IX $^{R}$ 
$\left(\mathrm{IX}^{H}\right)$ exhibit electron-hole pairs with parallel (antiparallel) spin-valley-locked magnetic moments. The emission from each IX species exhibits circular polarisation which, when combined with knowledge of the spin and valley configuration, enable determination of the atomic registry of the moiré trapping sites. We report the observation of a new moiré-trapping site for IX ${ }^{R}$ excitons in the TL-heterostructure as compared to IX ${ }^{R}$ excitons in $\mathrm{WSe}_{2} / \mathrm{MoSe}_{2}$ hetero-BLs 12, which we attribute to the $2 \mathrm{H}$-type stacking characteristic of $\mathrm{BL} \mathrm{MoSe}_{2}$ that results in the intrinsic locking of the atomic registries of the three layers. Our results present new evidence that add confidence to the moiré potential as the origin of the IX confinement.

\section{TRAPPED INTERLAYER EXCITONS IN A TRILAYER HETEROSTRUCTURE}

To justify our choice of TL heterostructure for the realisation of spin-layer locked IXs, we performed $a b$ initio calculations (see Suppl. Note 1). The artificial TL heterostructures consisted of a $2 \mathrm{H}-\mathrm{MoSe}_{2}$ crystal with ML and $\mathrm{BL}$ terraces mechanically stacked on top of a $\mathrm{WSe}_{2}$ ML and encapsulated by $\mathrm{hBN}$ in an inert environment (see Suppl. Note 2). The $2 \mathrm{H}$ stacking of $\mathrm{ML} \mathrm{WSe}_{2}$ and the bottom ML $\mathrm{MoSe}_{2}$ was confirmed by linearpolarisation-resolved second harmonic generation measurements (see Suppl Note 3). Figure 2 a shows representative low-temperature ( $\mathrm{T}=4 \mathrm{~K})$ confocal photoluminescence (PL) spectra measured using continuous wave excitation at $2.33 \mathrm{eV}$ and an excitation power $\left(P_{e x c}\right)$ of $4 \mu \mathrm{W}$. PL spectra corresponding to different positions of the $\mathrm{ML} \mathrm{MoSe}_{2} / \mathrm{ML} \mathrm{WSe} \mathrm{W}_{2}$ heterostructure (black and blue spectra) show emission in the energy range 1.32 $1.42 \mathrm{eV}$ (energy range I). We observe that the emission is centred around two main energy windows (1.385 - 1.405 $\mathrm{eV}$ and $1.345-1.375 \mathrm{eV}$ ), but with spatially dependent relative intensities. Positions A (black spectrum) and B (blue spectrum) correspond to the spatial positions with the brightest relative intensities for the two windows. To confirm that the observed emission arises from IXs, we performed PL excitation spectroscopy, scanning a continuous-wave excitation laser from $1.61 \mathrm{eV}$ to 1.75 $\mathrm{eV}$ while monitoring the intensity of the emission peaks. Figure $2 \mathrm{~b}$ shows a representative PL excitation spectrum, featuring two prominent resonances which correspond to the absorption of the intralayer $1 s$ exciton states in ML $\mathrm{MoSe}_{2}$ and $\mathrm{WSe}_{2}$. PL spectra in the TL part of the heterostructure is markedly different: emission in spectral region I is still observed but with reduced intensity relative to a new band of emission at lower energy (1.25 - 1.31 $\mathrm{eV}$, region II), see for example the red spectrum in Fig. $2 \mathrm{a}$. The spatial dependence of the PL spectra suggests that ranges I and II originate from different IX species, as expected from the stacking configuration presented in
Fig. 1. This heterostructure stacking configuration is corroborated by means of DC Stark effect measurements (see Suppl. Note 4). Therefore, we label IXs with emission in ranges I and II as $\mathrm{IX}^{H}$ and $\mathrm{IX}^{R}$, respectively. The PL emission energy of $\mathrm{IX}^{H}$ matches well with recent reports for IX emission in $\mathrm{MoSe}_{2} / \mathrm{WSe}_{2}$ hetero-BLs [12, 27, 29 31, while IX ${ }^{R}$ emission in TL heterostructures have yet to be explored in such detail.

With reducing $P_{e x c}$, the broad PL gradually disappears until the sharp peaks dominate, as shown in Suppl. Note 5. Here, high-resolution PL spectra measured with reduced $P_{\text {exc }}$ reveal that $\mathrm{IX}^{H}\left(\mathrm{IX}^{R}\right)$ PL peaks exhibit Lorentzian lineshapes with average full-width at half maximum (FWHM) of $\sim 100 \mu \mathrm{eV}(\sim 250 \mu \mathrm{eV})$; the narrowest peaks observed are $\sim 70 \mu \mathrm{eV}$ (see Suppl. Note $5)$. These linewidths, two orders of magnitude narrower than typical IX linewidths in $\mathrm{WSe}_{2} / \mathrm{MoSe}_{2}$ hetero-BLs 28 30, 32, are comparable to quantum emitters in ML $\mathrm{WSe}_{2}[33$ 35] and to recently reported moiré-trapped excitons [12]. Supplementary Figure 7 shows the full evolution of the $\mathrm{IX}^{H}$ emission under increasing $P_{\text {exc }}$. For each species of IX, the intensity saturates with increasing $P_{\text {exc }}$ according to a two-level system (Suppl. Fig. 7). Additionally, we observe minimal spectral wandering (< $10 \mu \mathrm{eV}$ ) at long time scales (see Suppl. Fig. 8).

\section{SPIN-VALLEY-LAYER CONFIGURATIONS OF TRAPPED INTERLAYER EXCITONS}

The narrow linewidths and saturation behaviour provide strong evidence of IXs trapped in a moiré confinement potential. For the low $P_{e x c}$ used in the PL spectra shown in Figs. 20-e, the IX density is not high enough to fill all the trapping sites of the moiré lattice within the diffraction limited focus of our confocal PL spot. Instead, we only observe emission from a few trapping sites (10 to 20 depending on the spatial position in the sample). Under increasing excitation power, as more trapping sites are filled, we progressively lose the ability to resolve individual spectral lines.

To confirm these peaks arise from band-edge states and disentangle the spin-layer-valley configuration of each exciton species, we perform magneto-optical spectroscopy measurements in Faraday configuration. Figure 3 a shows the magnetic field $\left(B_{z}\right)$ dependence of representative moiré-trapped IX ${ }^{R}$ and IX ${ }^{H}$ (left and right panel, respectively). A clear linear Zeeman splitting with increasing $B_{z}$ is observed for every peak, and it is immediately noticeable that the $g$-factor of IX $^{H}$ excitons is considerably larger than $\mathrm{IX}^{R}$ excitons. To confirm these are universal features of moiré trapped $\mathrm{IX}^{H}$ and $\mathrm{IX}^{R}$, a second $\mathrm{WSe}_{2} / \mathrm{MoSe}_{2}$ heterostructure with both $\mathrm{ML} \mathrm{WSe}_{2} / \mathrm{ML}$ $\mathrm{MoSe}_{2}$ and $\mathrm{ML} \mathrm{WSe}_{2} / \mathrm{BL} \mathrm{MoSe}_{2}$ regions and similar stacking angle $\left(\sim 60^{\circ}\right.$ between the $\left.\mathrm{ML} \mathrm{WSe}_{2} / \mathrm{BL} \mathrm{MoSe} \mathrm{Me}_{2}\right)$ was fabricated and measured (Sample 2). Sample 2 also 

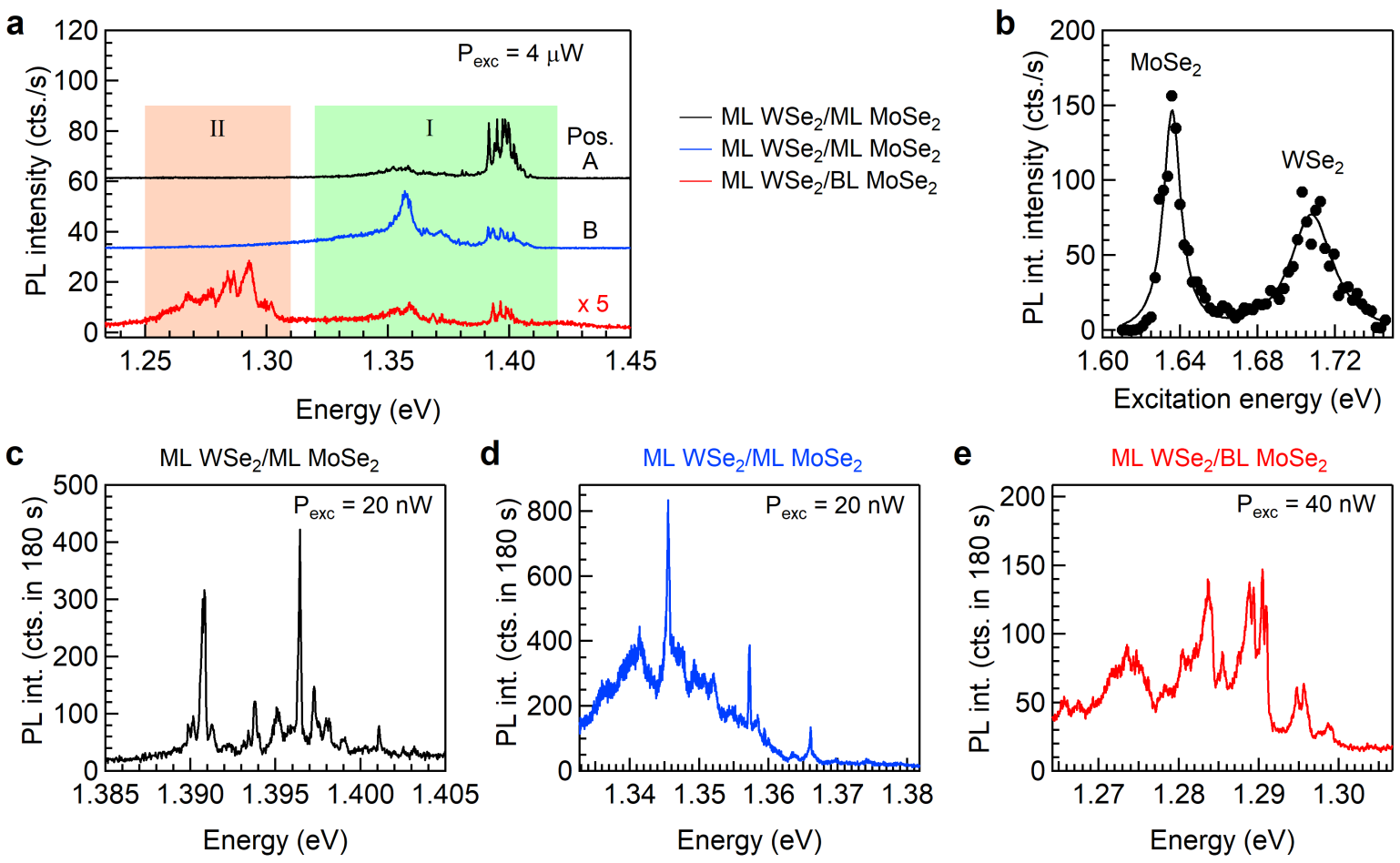

FIG. 2: Optical spectroscopy properties of spin-layer-locked IXs trapped in moiré potentials. a, Low-temperature confocal PL spectra measured at three spatial positions of the heterostructure. The black and blue positions (A and B, respectively) represent spectra corresponding to different positions of the ML $\mathrm{MoSe}_{2} / \mathrm{ML} \mathrm{WSe}_{2}$ heterostructure, with emission in energy range I: $1.32-1.42 \mathrm{eV}$ (green shaded area). The red line corresponds to a spectrum measured in the $\mathrm{ML}_{\mathrm{WSe}} / \mathrm{BL}$ $\mathrm{MoSe}_{2}$ region of the heterostructure, with emission in energy range II: $1.25-1.31 \mathrm{eV}$ (red shaded area). b, Photoluminescence excitation intensity plot of a representative IX (black dots), showing two resonances corresponding to the intralayer exciton states in $\mathrm{ML} \mathrm{MoSe} 2$ and $\mathrm{ML} \mathrm{WSe}{ }_{2}$. The black solid line represents a fit of the experimental data to two Lorentzian peaks. c-e, Optical spectra of representative IXs trapped in moiré potentials in the ML WSe $2 / \mathrm{ML} \mathrm{MoSe}_{2}$ (c and d), and the ML $\mathrm{WSe}_{2} / \mathrm{BL} \mathrm{MoSe}_{2}$ regions of the heterostructure (e) under low excitation power.

exhibits $\mathrm{IX}^{R}$ and $\mathrm{IX}^{H}$ with narrow emission peaks in the same energy ranges as Sample 1. Figure 3b summarises the measured $g$-values for each IX species in Samples 1 and 2 (black and red dots, respectively) as a function of their emission energy. Trapped $\mathrm{IX}^{R}$, which only appear in energy range II from the TL part of the samples, exhibit a $g$-factor of $-7.0 \pm 0.6$. On the other hand, trapped $\mathrm{IX}^{H}$ in energy range I are observed both in the hetero-BL and hetero-TL regions and exhibit a $g$-factor of $-15.76 \pm$ 0.13 .

The striking $B_{z}$ dependence for each IX species has its origin in their corresponding spin-valley configuration [12] (see Fig. 33), demonstrating that IX ${ }^{R}$ and IX ${ }^{H}$ preserve the spin-layer locking of electrons in BL $2 \mathrm{H}-\mathrm{MoSe}_{2}$. Since both carrier spin and valley index are associated to a magnetic moment [14, 15, the total valley-selective splitting of the interlayer transitions $(\Delta)$ amounts to [12, 36

$$
\Delta\left(B_{z}\right)=E^{\sigma^{+}}\left(B_{z}\right)-E^{\sigma^{-}}\left(B_{z}\right)=g \mu_{B} B_{z}
$$

with $g$ being an effective $g$-factor, $\mu_{B}$ being the Bohr magneton, and $E^{\sigma^{ \pm}}\left(B_{z}\right)$ being the $B_{z}$-dependent energies of the intervalley transitions with $\sigma^{ \pm}$polarisation. $E^{\sigma^{ \pm}}$is defined as the energy difference between the conduction $\left(E_{c}^{ \pm}\right)$and valence $\left(E_{v}^{ \pm}\right)$band edges associated to $\sigma^{ \pm}$transitions $\left(E^{\sigma^{ \pm}}\left(B_{z}\right)=E_{c}^{ \pm}-E_{v}^{ \pm}\right)$. The Zeeman shift of the $E_{c}^{ \pm}\left(\Delta E_{c}\right)$ and $E_{v}^{ \pm}\left(\Delta E_{v}\right)$ band edges can be estimated as a combination of three different magnetic moment contributions: $\Delta E_{c / v}=\Delta_{s}+\Delta_{a}+\Delta_{v}$, where $\Delta_{s}=2 s_{z} \mu_{B} B_{z}$ is the spin contribution, $\Delta_{a}=l_{c / v} \mu_{B} B_{z}$ is the atomic orbital contribution, and $\Delta_{v}=\tau \alpha_{c / v} \mu_{B} B_{z}$ represents the valley contribution arising from the Berry curvature [15]. Here, $s_{z}= \pm 1 / 2$ is the electron spin, $l_{c}=0\left(l_{v}=2 \tau\right)$ is the magnetic quantum number for the atomic orbital at the conduction (valence) band edge, $\tau= \pm 1$ is the index for the $\pm \mathrm{K}$ valleys, and $\alpha_{c}\left(\alpha_{v}\right)$ is the magnetic moment of the conduction (valence) band edge 18. According to the leading order of a simplified $\boldsymbol{k} \cdot \boldsymbol{p}$ approximation for the band-edge carriers [15, 37, the valley magnetic moments of the conduction and valence band can be estimated as $\alpha_{c, v}=m_{0} / m_{e, h}^{*}$, with $m_{0}$ the free electron mass and $m_{e}^{*}\left(m_{h}^{*}\right)$ the electron 
a

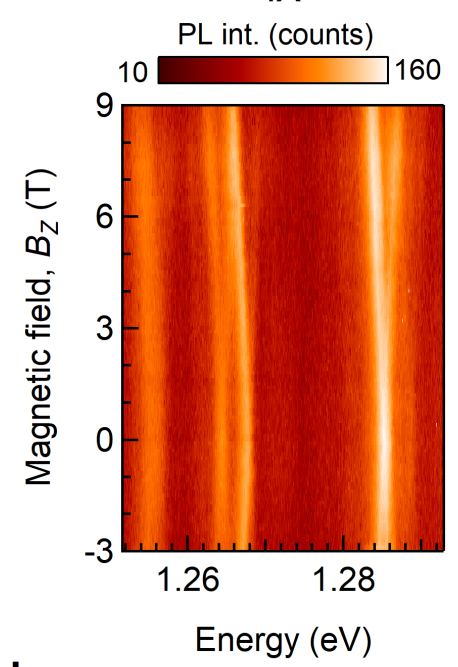

b

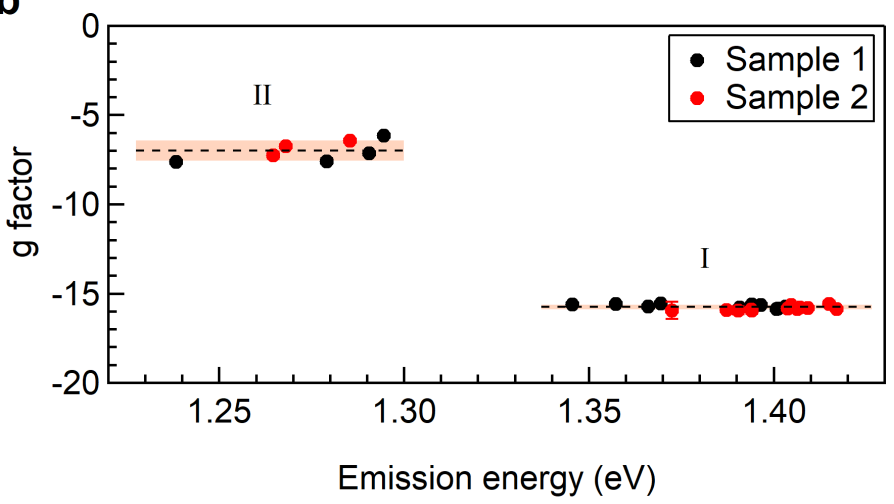

C
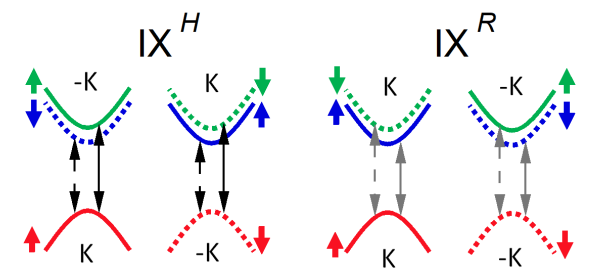

d
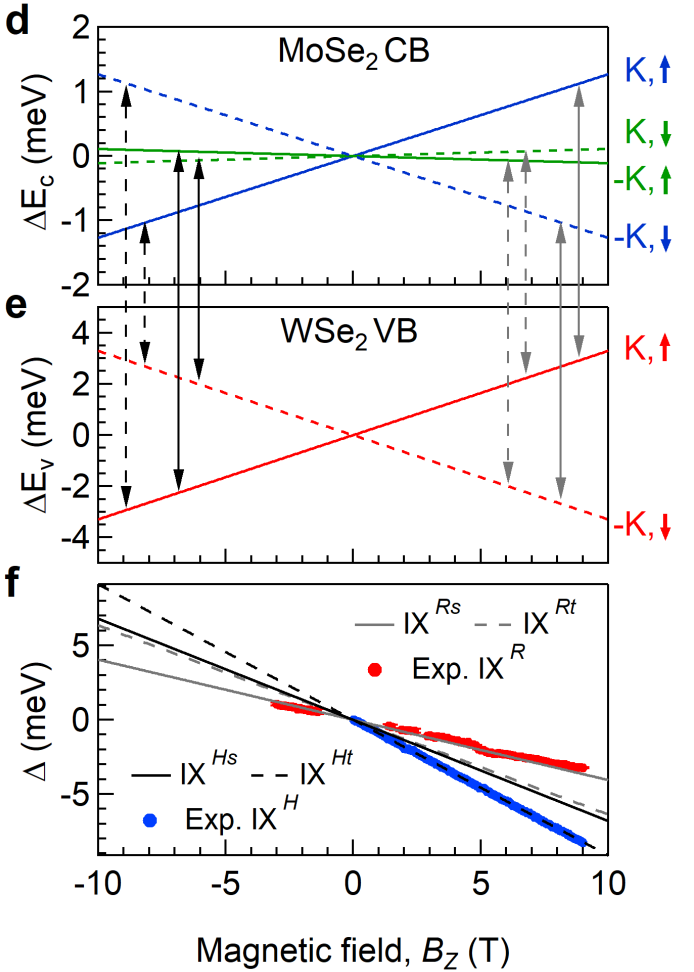

FIG. 3: Magneto-optic properties of spin-layer-locked IXs trapped in moiré potentials. a, Magnetic field dependence of representative moiré-trapped IX ${ }^{R}$ (left) and IX ${ }^{H}$ excitons (right) as a function of an applied out-of-plane magnetic field. $\mathbf{b}$, Summary of the $g$-factor values measured for moiré-trapped IX $^{R}$ and IX $^{H}$ excitons in heterostructures 1 (black dots) and 2 (red dots) as a function of their emission energies. The horizontal dashed lines and the shaded areas represent the average value and standard deviation of the $g$-factors, respectively. c, Schematics of the spin-valley configuration for IX ${ }^{R}$ (left) and IX ${ }^{H}$ (right) excitons. The vertical arrows represent the optical transitions for each IX exciton and spin configuration. d, e, Zeeman shifts for the conduction $\left(\Delta \mathrm{E}_{C}\right)$ and valence $\left(\Delta \mathrm{E}_{V}\right)$ band edges calculated by using Eqs. (2)-(5). The labels $\mathrm{K}(-\mathrm{K})$ and $\uparrow(\downarrow)$ represent the valley index and spin configuration of the corresponding band edges, respectively. Grey (black) arrows represent optical transitions in $\mathrm{IX}^{R}$ (IX ${ }^{H}$ ) excitons, while solid (dashed) lines represent spin-singlet (spin-triplet) optical transitions. f, $B_{z}$-dependence of the $\Delta$ value for for each IX exciton species and spin configuration as calculated from Eqs. $(2)-(5)$. IX ${ }^{H s}$, $\mathrm{IX}^{R s}, \mathrm{IX}^{H t}$ and IX ${ }^{R t}$, represent IXs arising from spin-singlet $(s)$ and spin-triplet $(t)$ optical transitions, respectively. The colour and line style used for the $\Delta$ value of each IX configuration is consistent the optical transitions of the corresponding excitons in $\mathbf{d}$ and $\mathbf{e}$. Red and blue dots represent the $B_{z}$-dependence of the experimental $\Delta$ values of representative trapped IX ${ }^{R}$ and $\mathrm{IX}^{H}$ excitons, respectively, as extracted from Lorentzian fits of the experimental data. Here the error bar size is smaller than the data points.

(hole) effective mass at the conduction (valence) band edge. Figures $3 \mathrm{~d}$ and $3 \mathrm{~s}$ show the Zeeman shifts of the conduction $\left(\mathrm{MoSe}_{2}\right)$ and valence $\left(\mathrm{WSe}_{2}\right)$ band edges of a $\mathrm{WSe}_{2} / \mathrm{MoSe}_{2}$ heterostructure, respectively, calculated using $m_{h}^{*}=0.37 m_{0}$ [38 and $m_{e}^{*}=0.84 m_{0}$ [39, 40. Here we assume that the spin-split conduction bands at the $\pm \mathrm{K}$ points of $\mathrm{ML}$ and $\mathrm{BL} \mathrm{MoSe}_{2}$ have similar effective masses 39, and therefore we use the same $\alpha_{c}$ for both. The colour and line style used for each band edge are consistent with the schematics of the spin-layer configuration shown in Fig. 33. Solid (dashed) lines represent the Zeeman shifts of band edges with spin up (down). Red lines represent the Zeeman shifts of the valence band states in $\mathrm{WSe}_{2}$, whereas blue and green lines represent the Zeeman shifts of the $\mathrm{MoSe}_{2}$ conduction band edges with parallel and antiparallel valley and spin configuration, respectively.

According to Equation (1), the different spin-layer- 
valley configuration of $\mathrm{IX}^{R}$ and $\mathrm{IX}^{H}$ excitons results in different total Zeeman splittings $\Delta$. Therefore, the $g$ factor of the moiré-trapped valley excitons is representative of their spin-valley configuration, which, as a consequence of the spin-layer locking of electrons in $\mathrm{BL} \mathrm{MoSe}$, also indicates whether the electron is localised in the bottom or top $\mathrm{MoSe}_{2}$ layer. Furthermore, two different $\Delta$ splittings are possible for both IX ${ }^{R}$ and IX ${ }^{H}$ excitons depending on the conduction bands involved in the optical transitions (see Methods).

The vertical arrows in Figs. $3 \mathrm{~d}$ and 3 e represent the optical transitions responsible of the Zeeman splittings for each IX exciton and spin configuration. Grey (black) arrows represent optical transitions in $\operatorname{IX}^{R}\left(\mathrm{IX}^{H}\right)$ excitons, while solid (dashed) lines represent spin-singlet (spin-triplet) optical transitions. Figure 3f shows the $B_{z}$-dependence of the $\Delta$ value for each IX exciton and spin configuration as calculated from Equations (2)-(5). The colour and line style used for the $\Delta$ value of each IX configuration is consistent with the one employed to indicate the optical transitions of the corresponding excitons. Figure 3 also shows the $B_{z}$-dependence of the experimental $\Delta$ values of two representative trapped IX $^{R}$ (red dots) and $\mathrm{IX}^{H}$ (blue dots) excitons, as extracted from Lorentzian fits of the experimental data. The good agreement observed between the calculated and experimental $\Delta$ values corroborates our initial identification of $\mathrm{IX}^{R}$ and $\mathrm{IX}^{H}$ excitons. Moreover, the magneto-optical measurements provide additional information. The results shown in Fig. 3: indicate that the observed $\mathrm{IX}^{R}$ and $\mathrm{IX}^{H}$ excitons arise from optical transitions involving the lowest spin-split conduction band of $\mathrm{MoSe}_{2}$ at $\pm \mathrm{K}$. This observation leads to spin-conserved and spin-flip optical transitions for IX ${ }^{R}$ and IX $^{H}$ excitons, respectively. Although the latter is normally forbidden in ML TMDs due to its spin-flip nature, it can be brightened due to the selection rules dictated by the resulting interlayer atomic registry of the moiré pattern in our heterostrtuctures [11].

The spin-layer-locked nature of $\mathrm{IX}^{R}$ and $\mathrm{IX}^{H}$ allows us to estimate the magnetic moment contribution of the conduction and valence band edges, and therefore the corresponding carrier effective masses (see Methods).

\section{LOCAL ATOMIC REGISTRIES OF MOIRÉ TRAPPING SITES}

The relative Zeeman shifts of the conduction and valence band edges at $\pm \mathrm{K}$ points enable further insight, based on the fact that the $B_{z}$-induced shift of the $\mathrm{WSe}_{2}$ valence band edge is larger than the corresponding shifts of the $\mathrm{MoSe}_{2}$ conduction bands (as shown in Figs. $3 \mathrm{~d}$ and 3 ). The smaller Zeeman splitting for the conduction band edges is a consequence of two factors: the smaller magnetic moment contribution from the atomic orbitals of the conduction band $\left(l_{c}=0\right)$, and the higher effective mass of electrons in the conduction band of $\mathrm{MoSe}_{2}$. The larger Zeeman shift for the valence band edges leads to a striking consequence: for $B_{z}>0$, the energy of the optical transitions involving the valence band edges of $\mathrm{WSe}_{2}$ at $\mathrm{K}(-\mathrm{K})$ always shift to lower (higher) energies regardless of the spin-valley configuration of the IX. Based on this, helicity-resolved PL measurements can provide information about the nature of the moiré confinement potential. Figures 4 a and $4 \mathrm{p}$ show circularly-polarised-resolved spectra of representative IX ${ }^{H}$ and IX ${ }^{R}$, respectively, under linearly polarised $(\pi)$ excitation at $2.33 \mathrm{eV}$ and different applied magnetic fields. Both IX $^{H}$ and IX ${ }^{R}$ exhibit strong circular polarisation, and application of $B_{z}$ results in a Zeeman shift of the $\sigma^{+}$-polarised ( $\sigma^{-}$-polarised) PL peak towards lower (higher) energies. For zero magnetic field, energydegenerate $\sigma^{+}$and $\sigma^{-}$emissions are observed with near identical intensity, indicating that the IXs are trapped in confinement potentials that preserve the rotational $C_{3}$ symmetry. The degenerate circularly-polarised emission of IX ${ }^{R}$ and IX $^{H}$ contrasts with the emission polarisation properties of neutral excitons in quantum emitters in ML $\mathrm{WSe}_{2}$, which typically exhibit a large fine-structure splitting and strictly linear polarisation [33 35 ] arising from the electron-hole exchange interaction energy and asymmetry in the confinement potential. The absence of observable fine-structure splitting is only expected in cases for which rotational symmetry of the crystal lattice and confining potential is maintained.

The polarisation selection rules of the moiré-trapped excitons are dictated by the local atomic registry of the moiré trapping site [9, 11]. Figure 44 shows the selection rules for optical transitions involving the K-point valence band for both spin-singlet and spin-triplet IXs trapped in moiré potential sites with different atomic registries [11]. Accordingly, the results in Figs. $4 \mathrm{a}$ and $4 \mathrm{~b}$ indicate that IX $^{R}$ and IX $^{H}$ originate from IXs trapped in moiré potentials with interlayer atomic registries $R_{h}^{h}(\mathrm{~A})$ and $H_{h}^{h}$ (B), respectively. Interestingly, we find that IX ${ }^{R}$ excitons in the TL heterostructure present a comparable $g$-factor magnitude but opposite valley selection rules compared to $\mathrm{IX}^{R}$ excitons in $\mathrm{WSe}_{2} / \mathrm{MoSe}_{2}$ hetero-BLs 12. These results indicate that localised $\mathrm{IX}^{R}$ excitons in the TL heterostructure and in $\mathrm{WSe}_{2} / \mathrm{MoSe}_{2}$ hetero-BLs [12] are the same IX species (same spin-valley pairing) trapped in moiré potentials with different atomic registries. We attribute the different atomic registry of the moiré trapping potentials for IX ${ }^{R}$ excitons in the TL heterostructure $\left(R_{h}^{h}\right)$ and the $\mathrm{WSe}_{2} / \mathrm{MoSe}_{2}$ hetero-BLs $\left(R_{h}^{X}\right)$ to the $2 \mathrm{H}$-type stacking of characteristic of $\mathrm{BL} \mathrm{MoSe}_{2}$, which results in the intrinsic locking of the atomic registries of the three layers (see left panel of Fig. 44). 
a

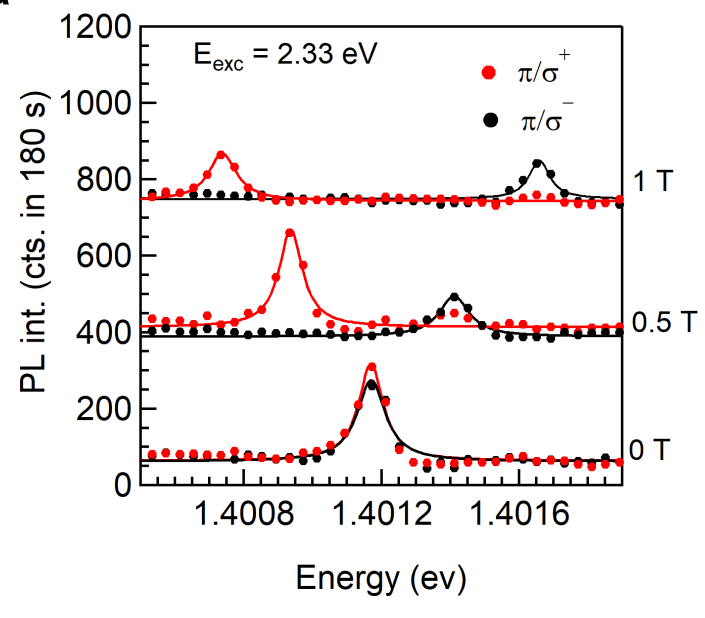

b

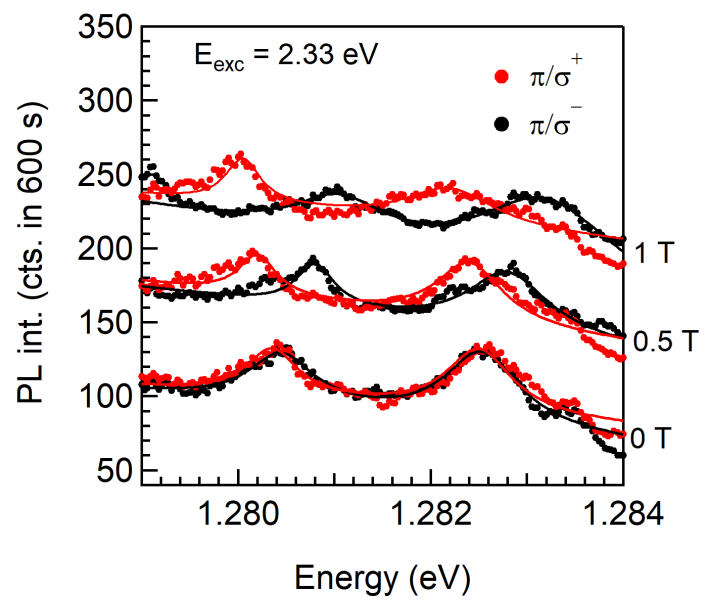

C

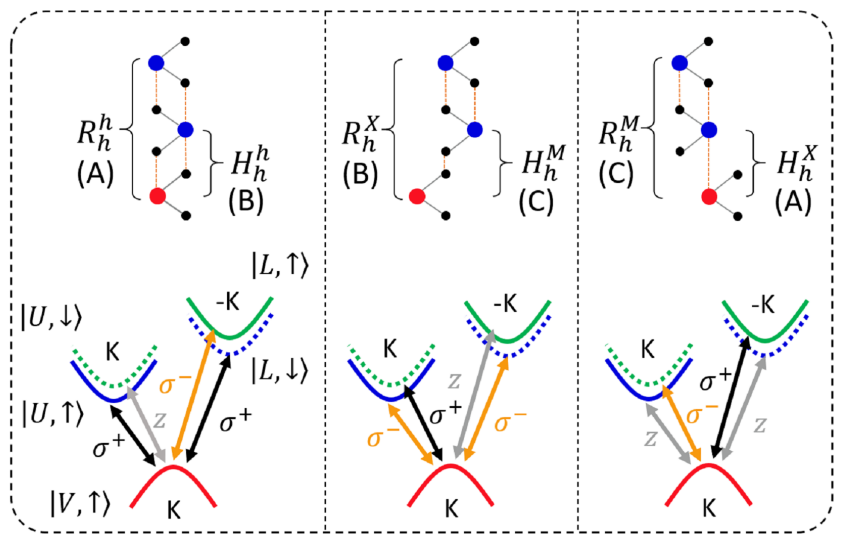

FIG. 4: Optical selection rules of spin-layer-locked IXs trapped in moiré potentials. a-b, PL spectra of representative $\mathrm{IX}^{H}$ and $\mathrm{IX}^{R}$ excitons, respectively, under linearly polarised excitation at $2.33 \mathrm{eV}$ and different applied magnetic fields for circularly-polarised-resolved $\sigma^{+}$(red dots) and $\sigma^{-}$(back dots) collection. The red and black solid lines represent Lorentzian fits to the experimental data. c, Schematics of the selection rules for optical transitions involving the K-point valence band for both spin-singlet and spin-triplet IXs trapped in moiré potential sites with different atomic registries [1]. Black, orange and grey double arrows represent optical transitions with $\sigma^{+}, \sigma^{-}$, and $z$ polarisation. $R(H)_{h}^{\mu}$ denotes an $R(H)$-type stacking with the $\mu$ site of the electron layer (either $h$ the hexagon centre, $X$ the chalcogen site or $M$ the metal site) vertically aligned with the hexagon centre $(h)$ of the hole layer.

\section{DISCUSSION AND OUTLOOK}

The magneto-optical spectroscopy experiments reveal the remarkable consequence of spin-layer locking of moiré-trapped excitons in the artificial TL heterostructures. Two IX species, IX ${ }^{R}$ and $\mathrm{IX}^{H}$, with distinct spin-valley-layer configurations are observed: $\mathrm{IX}^{R}\left(\mathrm{IX}^{H}\right)$ composed of electrons in the top (bottom) $\mathrm{MoSe}_{2}$ layer present carriers with parallel (antiparallel) spin-valley locked contributions, resulting in an effective layerlocking of the Landé g-factors of the trapped IX. At cryogenic temperatures, both trapped IX species exhibit narrow linewidths and saturate with increasing excitation power, hallmarks of a few-level quantum confined system. An unambiguous demonstration of quantum emis- sion from the moiré-trapped excitons, for instance photon antibunching, remains an important target. Finally, the combination of magneto-optics and helicity-resolved PL measurements allows the determination of the atomic registries of the moiré trapping sites.

For quantum information applications, a basic requirement is the ability to initialise the state of the fewlevel quantum system, which has been achieved for valley qubits in ML TMDs via optical pumping [16, 17]. Here we show the trapped IXs retain the strong valley polarisation of the constituent ML semiconductors (see Suppl. Fig. 10).

Finally, we remark that the phenomenon of spin-layer locking provides a means to engineer few-level quantum systems in van der Waals heterostructures. For example, the selection rules arising from the $C_{3}$ symmetry for the 
atomic registries we observe (left panel of of Fig. 44), create a "vee-type" three level system: a single ground state $(|V, \uparrow\rangle)$ couples to two non-degenerate excited states $(|L, \downarrow\rangle$ and $|U, \uparrow\rangle)$. Alternatively, a three-level system analogous to a "spin-lambda" type atom, in which two ground states couple to a common excited state, can be engineered using the layer-index degree of freedom in a TL heterostructure consisting of BL $2 \mathrm{H}-\mathrm{WSe}_{2} / \mathrm{ML}$ $\mathrm{MoSe}_{2}$. This quantum state engineering opens new opportunities for advanced quantum control techniques in the van der Waals platform.

* These authors contributed equally to this work; Electronic address: M.Brotons ïGisbert@hw.ac.uk; h.baek@hw.ac.uk

$\dagger$ Electronic address: B.D.Gerardot@hw.ac.uk

[1] Geim, A. K. \& Grigorieva, I. V. Van der waals heterostructures. Nature 499, 419 (2013).

[2] Bistritzer, R. \& MacDonald, A. H. Moiré bands in twisted double-layer graphene. Proc. Natl. Acad. Sci. 108, 12233-12237 (2011).

[3] Zhang, C. et al. Interlayer couplings, Moiré patterns, and $2 \mathrm{D}$ electronic superlattices in $\mathrm{MoS}_{2} / \mathrm{WSe}_{2}$ heterobilayers. Sci. Adv. 3, e1601459 (2017).

[4] Cao, Y. et al. Unconventional superconductivity in magic-angle graphene superlattices. Nature 556, 43 (2018).

[5] Jin, C. et al. Observation of moiré excitons in $\mathrm{WSe}_{2} / \mathrm{WS}_{2}$ heterostructure superlattices. Nature 567, 76 (2019).

[6] Alexeev, E. M. et al. Resonantly hybridized excitons in moiré superlattices in van der Waals heterostructures. Nature 567, 81 (2019).

[7] Tong, Q. et al. Topological mosaics in moiré superlattices of van der Waals heterobilayers. Nat. Phys. 13, 356 (2017).

[8] Yankowitz, M. et al. Dynamic band-structure tuning of graphene moiré superlattices with pressure. Nature 557, 404 (2018).

[9] Yu, H., Liu, G.-B., Tang, J., Xu, X. \& Yao, W. Moiré excitons: From programmable quantum emitter arrays to spin-orbit-coupled artificial lattices. Sci. Adv. $\mathbf{3}$, e1701696 (2017).

[10] Wu, F., Lovorn, T. \& MacDonald, A. Theory of optical absorption by interlayer excitons in transition metal dichalcogenide heterobilayers. Phys. Rev. B 97, 035306 (2018).

[11] Yu, H., Liu, G.-B. \& Yao, W. Brightened spin-triplet interlayer excitons and optical selection rules in van der waals heterobilayers. 2D Materials 5, 035021 (2018).

[12] Seyler, K. L. et al. Signatures of moiré-trapped valley excitons in $\mathrm{MoSe}_{2} / \mathrm{WSe}_{2}$ heterobilayers. Nature 567, 6670 (2019).

[13] Tran, K. et al. Evidence for moiré excitons in van der Waals heterostructures. Nature 1 (2019).

[14] Xu, X., Yao, W., Xiao, D. \& Heinz, T. F. Spin and pseudospins in layered transition metal dichalcogenides. Nat. Phys. 10, 343 (2014).

[15] Xiao, D., Liu, G.-B., Feng, W., Xu, X. \& Yao, W. Coupled spin and valley physics in monolayers of $\mathrm{MoS}_{2}$ and other group-VI dichalcogenides. Phys. Rev. Lett. 108, 196802 (2012).

[16] Mak, K. F., He, K., Shan, J. \& Heinz, T. F. Control of valley polarization in monolayer $\mathrm{MoS}_{2}$ by optical helicity. Nat. Nanotechnol. 7, 494 (2012).

[17] Zeng, H., Dai, J., Yao, W., Xiao, D. \& Cui, X. Valley polarization in $\mathrm{MoS}_{2}$ monolayers by optical pumping. Nat. Nanotechnol. 7, 490 (2012).

[18] Aivazian, G. et al. Magnetic control of valley pseudospin in monolayer $\mathrm{WSe}_{2}$. Nat. Phys. 11, 148 (2015).

[19] Srivastava, A. et al. Valley Zeeman effect in elementary optical excitations of monolayer $\mathrm{WSe}_{2}$. Nat. Phys. 11, 141 (2015).

[20] Gong, Z. et al. Magnetoelectric effects and valleycontrolled spin quantum gates in transition metal dichalcogenide bilayers. Nat. Commun. 4, 2053 (2013).

[21] Jones, A. M. et al. Spin-layer locking effects in optical orientation of exciton spin in bilayer $\mathrm{WSe}_{2}$. Nat. Phys. 10, 130 (2014).

[22] Riley, J. M. et al. Direct observation of spin-polarized bulk bands in an inversion-symmetric semiconductor. Nat. Phys. 10, 835 (2014).

[23] Chiu, M.-H. et al. Determination of band alignment in the single-layer $\mathrm{MoS}_{2} / \mathrm{WSe}_{2}$ heterojunction. Nat. Commun. 6, 7666 (2015).

[24] Wilson, N. R. et al. Determination of band offsets, hybridization, and exciton binding in 2D semiconductor heterostructures. Sci. Adv. 3, e1601832 (2017).

[25] Hong, X. et al. Ultrafast charge transfer in atomically thin $\mathrm{MoS}_{2} / \mathrm{WS}_{2}$ heterostructures. Nat. Nanotechnol. 9, 682 (2014).

[26] Yu, H., Wang, Y., Tong, Q., Xu, X. \& Yao, W. Anomalous light cones and valley optical selection rules of interlayer excitons in twisted heterobilayers. Phys. Rev. Lett. 115, 187002 (2015).

[27] Rivera, P. et al. Observation of long-lived interlayer excitons in monolayer $\mathrm{MoSe}_{2}-\mathrm{WSe}_{2}$ heterostructures. Nat. Commun. 6, 6242 (2015).

[28] Rivera, P. et al. Valley-polarized exciton dynamics in a 2D semiconductor heterostructure. Science 351, 688-691 (2016).

[29] Hanbicki, A. T. et al. Double indirect interlayer exciton in a $\mathrm{MoSe}_{2} / \mathrm{WSe}_{2}$ van der Waals heterostructure. Acs Nano 12, 4719-4726 (2018).

[30] Ciarrocchi, A. et al. Polarization switching and electrical control of interlayer excitons in two-dimensional van der Waals heterostructures. Nat. Photonics 13, 131-136 (2019).

[31] Torun, E., Miranda, H. P. C., Molina-Sánchez, A. \& Wirtz, L. Interlayer and intralayer excitons in $\mathrm{MoS}_{2} / \mathrm{WS}_{2}$ and $\mathrm{MoSe}_{2} / \mathrm{WSe}_{2}$ heterobilayers. Phys. Rev. $B$ 97, 245427 (2018).

[32] Jiang, C. et al. Microsecond dark-exciton valley polarization memory in two-dimensional heterostructures. Nat. Commun. 9, 753 (2018).

[33] Srivastava, A. et al. Optically active quantum dots in monolayer $\mathrm{WSe}_{2}$. Nat. Nanotechnol. 10, 491 (2015).

[34] Tonndorf, P. et al. Single-photon emission from localized excitons in an atomically thin semiconductor. Optica $\mathbf{2}$, 347-352 (2015).

[35] Brotons-Gisbert, M. et al. Coulomb blockade in an atomically thin quantum dot coupled to a tunable fermi reservoir. Nat. Nanotechnol. 14, 442-446 (2019).

[36] Nagler, P. et al. Giant magnetic splitting inducing near- 
unity valley polarization in van der Waals heterostructures. Nat. Commun. 8, 1551 (2017).

[37] Yao, W., Xiao, D. \& Niu, Q. Valley-dependent optoelectronics from inversion symmetry breaking. Phys. Rev. B 77, 235406 (2008).

[38] Kormányos, A. et al. k·p theory for two-dimensional transition metal dichalcogenide semiconductors. 2D Materials 2, 022001 (2015).

[39] Larentis, S. et al. Large effective mass and interactionenhanced Zeeman splitting of K-valley electrons in $\mathrm{MoSe}_{2}$. Phys. Rev. B 97, 201407 (2018).

[40] Goryca, M. et al. Revealing exciton masses and dielectric properties of monolayer semiconductors with high magnetic fields. Nat. Commun. 10, 1-12 (2019).

\section{ACKNOWLEDGEMENTS}

The authors thank J. J. Finley, K. Müller, M. Kremser, and A. Högele for discussions. This work is supported by the EPSRC (grant no. EP/P029892/1 and $\mathrm{EP} / \mathrm{S} 000550 / 1$ ), the ERC (grant no. 725920) and the EU Horizon 2020 research and innovation program under grant agreement no. 820423. AMS acknowledges the Juan de la Cierva (Grant IJCI-2015-25799) program (MINECO, Spain) and the Marie-Curie-COFUND program Nano TRAIN For Growth II (Grant Agreement 713640). The computations were performed on the Tirant III cluster of the Servei d'Informàtica of the University of Valencia (project vlc82). Growth of hBN crystals by K.W. and T.T. was supported by the Elemental Strategy Initiative conducted by the MEXT, Japan and the CREST (JPMJCR15F3). B.D.G. is supported by a Wolfson Merit Award from the Royal Society and a Chair in Emerging Technology from the Royal Academy of Engineering.

\section{AUTHOR CONTRIBUTIONS}

B.D.G. conceived and supervised the project. H.B. fabricated the samples. K.W. and T.T. supplied the hBN crystals. M.B.-G. and H.B. performed the experiments, assisted by A.C., C.B. and D.W. M.B.-G. analysed the data, assisted by E.S. and B.D.G. A.M.-S. performed the ab initio calculations. M.B.-G. and B.D.G. cowrote the paper with input from all authors. M.B.-G. and H.B. contributed equally to this work.

\section{COMPETING INTERESTS}

The authors declare no competing interests. 


\section{METHODS}

\section{Zeeman splitting for IXs with different spin-layer-valley configurations}

Optical transitions between the bottom conduction band states and the top valence band state at $K$ give rise to four possible IX configurations: $\operatorname{IX}^{H s}(|V, \uparrow\rangle \leftrightarrow|L, \uparrow\rangle)$, $\operatorname{IX}^{H t}(|V, \uparrow\rangle \leftrightarrow|L, \downarrow\rangle), \operatorname{IX}^{R s}(|V, \uparrow\rangle \leftrightarrow|U, \uparrow\rangle)$, and IX ${ }^{R t}$ $(|V, \uparrow\rangle \leftrightarrow|U, \downarrow\rangle)$, where $s$ and $t$ superscripts denote spinconserving (spin-singlet) and spin-flip (spin-triplet) optical transitions, respectively.

Using Eq. (1), IX ${ }^{R}$ excitons can take $\Delta$ values of:

$$
\left|\Delta E^{R s}\left(B_{z}\right)\right|=2\left|2+\alpha_{v}-\alpha_{c}\right| \mu_{B} B_{z},
$$

and

$$
\left|\Delta E^{R t}\left(B_{z}\right)\right|=2\left|4+\alpha_{v}-\alpha_{c}\right| \mu_{B} B_{z} ;
$$

while $\mathrm{IX}^{H}$ excitons can present $\Delta$ values of:

$$
\left|\Delta E^{H s}\left(B_{z}\right)\right|=2\left|2+\alpha_{v}+\alpha_{c}\right| \mu_{B} B_{z},
$$

and

$$
\left|\Delta E^{H t}\left(B_{z}\right)\right|=2\left|4+\alpha_{v}+\alpha_{c}\right| \mu_{B} B_{z} .
$$

\section{Magnetic moment contributions of the band edges}

From the combination of Equations (2) and (5), and the measured $g$-factors for IX $^{H}$ and IX ${ }^{R}$, we estimate magnetic moment contributions of $\alpha_{v}=2.69 \pm 0.15$ and $\alpha_{c}=1.19$ \pm 0.15 . The estimated magnetic moment for the band edges yield effective masses of $m_{e}^{*} \approx 0.84 \pm 0.11 m_{0}$ and $m_{h}^{*} \approx 0.37 \pm$ $0.02 m_{0}$ for electrons and holes at the bottom conduction band of $\mathrm{MoSe}_{2}$ and top valence band of $\mathrm{WSe}_{2}$, respectively.

In the previous discussion we have considered that $\mathrm{IX}^{H}$ arise from optical spin-flip transitions involving the lowest spin-split conduction band of $\mathrm{MoSe}_{2}$ at $\pm \mathrm{K}$. However, IX ${ }^{H}$ could also be tentatively attributed to spin-conserved optical transitions from the top valence band of $\mathrm{WSe}_{2}$ and the higher energy spin-split conduction band of $\mathrm{MoSe}_{2}$ at $\pm \mathrm{K}$ [12] (see Fig. 3c). For such spin-singlet configuration $\left(\mathrm{IX}^{H s}\right)$, the spin magnetic moments contribution of the electron and hole cancel each other, and the total Zeeman splitting can be calculated by Eq. (4). In this case, the combination of the measured $g$-factors with Eqs. (2) and (4) leads to effective masses of $m_{e}^{*} \approx 0.46 \pm 0.03 m_{0}$ and $m_{h}^{*} \approx 0.27 \pm 0.01$ $m_{0}$ for electrons and holes at the top conduction band of $\mathrm{MoSe}_{2}$ and top valence band of $\mathrm{WSe}_{2}$, respectively. These values differ significantly from the corresponding reported effective masses. On the other hand, assuming a spin-triplet configuration for IX $^{H}$ results in estimated carrier effective masses that match well previously calculated and experimental values 38 40, providing confidence that both trapped $\mathrm{IX}^{H}$ and $\mathrm{IX}^{R}$ originate from optical transitions involving the lowest split conduction band of $\mathrm{MoSe}_{2}$ at $\pm \mathrm{K}$.

\section{DATA AVAILABILITY}

Data described in this paper and presented in the Supplementary materials are available online at https://researchportal.hw.ac.uk/en/persons/brian-dgerardot/datasets/ 